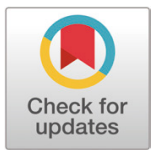

Received: Mar 18, 2020

Revised: Mar 19, 2020

Accepted: Mar 19, 2020

\#These authors contributed equally to this work.

*Corresponding author

Younghoon Kim

Department of Agricultural

Biotechnology and Research Institute

of Agriculture and Life Science,

Seoul National University,

Seoul 08826, Korea

Tel: +82-2-880-4808

E-mail: ykeys2584@snu.ac.kr

Sangnam Oh

Department of Functional Food and

Biotechnology, Jeonju University,

Jeonju 55069, Korea.

Tel: +82-63-220-3109

E-mail:osangnam@jj.ac.kr

Copyright $\odot 2020$ Korean Society of Animal Sciences and Technology.

This is an Open Access article distributed under the terms of the Creative Commons Attribution

Non-Commercial License (http:// creativecommons.org/licenses/by$\mathrm{nc} / 4.0 /$ ) which permits unrestricted non-commercial use, distribution, and

\section{Complete chromosome and plasmid sequences of Staphylococcus au- reus strain JDFM SA01, isolated from a milk filter in Korean dairy farm}

\author{
Sangdon Ryu', Donghyun Shin ${ }^{2}$, Jaeyoung Heo ${ }^{3}$, Seong-Yeop Jeong ${ }^{4}$, \\ Do-Youn Jeong ${ }^{4}$, Bohyun Yun ${ }^{1}$, Minkyoung Kang ${ }^{5}$, Younghoon Kim ${ }^{6 * *}$ \\ and Sangnam $\mathrm{Oh}^{5 \# *}$ \\ ${ }^{1}$ Department of Animal Science and Institute of Milk Genomics, Jeonbuk National University, Jeonju \\ 54896, Korea \\ ${ }^{2}$ The Animal Molecular Genetics and Breeding Center, Jeonbuk National University, Jeonju 54896, Korea \\ ${ }^{3}$ International Agricultural Development and Cooperation Center, Jeonbuk National University, Jeouju \\ 54896, Korea \\ ${ }^{4}$ Microbial Institute for Fermentation Industry, Sunchang 56048, Korea \\ ${ }^{5}$ Department of Functional Food and Biotechnology, Jeonju University, Jeonju 55069, Korea \\ ${ }^{6}$ Department of Agricultural Biotechnology and Research Institute of Agriculture and Life Science, Seoul \\ National University, Seoul 08826, Korea
}

\section{Abstract}

Staphylococcus aureus is a significant pathogen that can source a variety of illness worldwide. In this announcement, we report here the complete genome sequence of $S$. aureus strain JDFM SA01, isolated from a milk filter collected from Korean dairy farm. The final complete genome assembly consists of one circular chromosome $(2,748,925 \mathrm{bp})$ with an overall GC content of $32.9 \%$ and one circular plasmid sequence $(24,655 \mathrm{bp})$ with a GC content of $28.7 \%$.

Keywords: Staphylococcus aureus strain JDFM SA01, Dairy farm, Whole genome sequencing

Staphylococcus aureus is a Gram-positive bacteria that is detected in the environment and also existing in normal human microbiome [1]. In addition, this bacterium is a representative bacterial human pathogen and contaminated with food through purulent wounds of humans and animals. Particularly, methicillin-resistant $S$. aureus (MRSA) has appeared as a significant issue, with important anxieties about public health because they can spread easily through healthy carriers and increase the likelihood of in-

Table 1. Genome features of Staphylococcus aureus strain JDFM SA01

\begin{tabular}{lrrrrrr}
\hline \multicolumn{1}{c}{ Name } & Length (bp) & GC (\%) & Depth & CDSs & tRNA & rRNA \\
\hline Chromosome & $2,748,925$ & 32.9 & 408 & 2,520 & 61 & 19 \\
Plasmid & 24,655 & 28.7 & 323 & 29 & 0 & 0 \\
Total & $2,773,580$ & 32.84 & 407 & 2,549 & 61 & 19 \\
\hline
\end{tabular}

CDSs, coding DNA sequences. 
reproduction in any medium, provided the original work is properly cited.

ORCID

Sangdon Ryu

https://orcid.org/0000-0001-5338-8385

Dong Hyun Shin

https://orcid.org/0000-0002-0819-0553

Jae Young Heo

https://orcid.org/0000-0002-9721-8043

Seong-Yeop Jeong

https://orcid.org/0000-0001-8995-9901

Do-Youn Jeong

https://orcid.org/0000-0003-4105-1624

Bohyun Yun

https://orcid.org/0000-0001-6723-5849

Min Kyoung Kang

https://orcid.org/0000-0002-2366-7970

Younghoon Kim

https://orcid.org/0000-0001-6769-0657

Sangnam Oh

https://orcid.org/0000-0002-2428-412X

\section{Competing interests}

No potential conflict of interest relevant to

this article was reported.

Funding sources

This research was supported by the High Value-Added Food Technology Development Program of the Korean Institute of Planning and Evaluation for Technology in Food, Agriculture, Forestry, and Fisheries (iPET), the Ministry for Food, Agriculture, Forestry, and Fisheries of the Korea (318090-03-1-WT011) and Microbial Institute for Fermentation Industry (MIFI) through Establishment of Integrated Biobank for Agriculture, Food and Livestock Microbiome Project, funded by Ministry of Agriculture, Food and Rural Affairs (MAFRA) (MIFI2020KMBB01)

Acknowledgements

Not applicable.

Availability of data and material

The complete genome sequences have been deposited in GenBank under the accession numbers CP032821 and CP032822 for the $S$. aureus strain JDFM SA01 chromosome and plasmid, respectively. The BioProject accession number is PRJNA491802, and the BioSample accession numbers are SAMN10147904 for S. aureus strain JDFM SA01.

Authors' contributions

Conceptualization: Ryu S, Kim Y, Oh S.

Data curation: Ryu S, Shin D, Kim Y, Oh S.

Formal analysis: Ryu S, Shin D, Kim Y, Oh S.

Methodology: Ryu S, Kim Y, Oh S.

Software: Ryu S, Shin D, Kim Y, Oh S.

Validation: Ryu S, Kim Y, Oh S.

Investigation: Kim Y, Oh S.

Writing - original draft: Ryu S, Shin D, Heo J, Jeong SY, Jeong DY, Yun B, Kang M, Kim Y, Oh S.

Writing - review \& editing: Ryu S, Shin D, Heo J, Jeong SY, Jeong DY, Yun B, Kang M, Kim Y, Oh S.
(A)

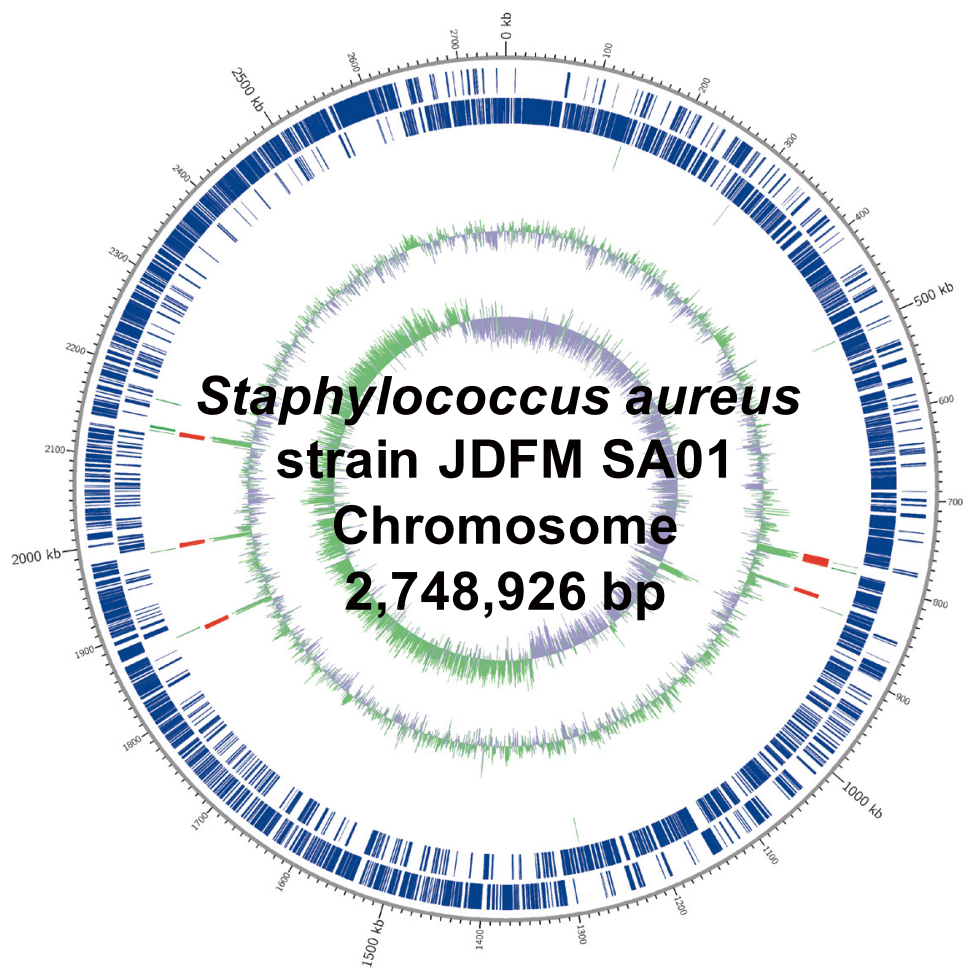

(B)

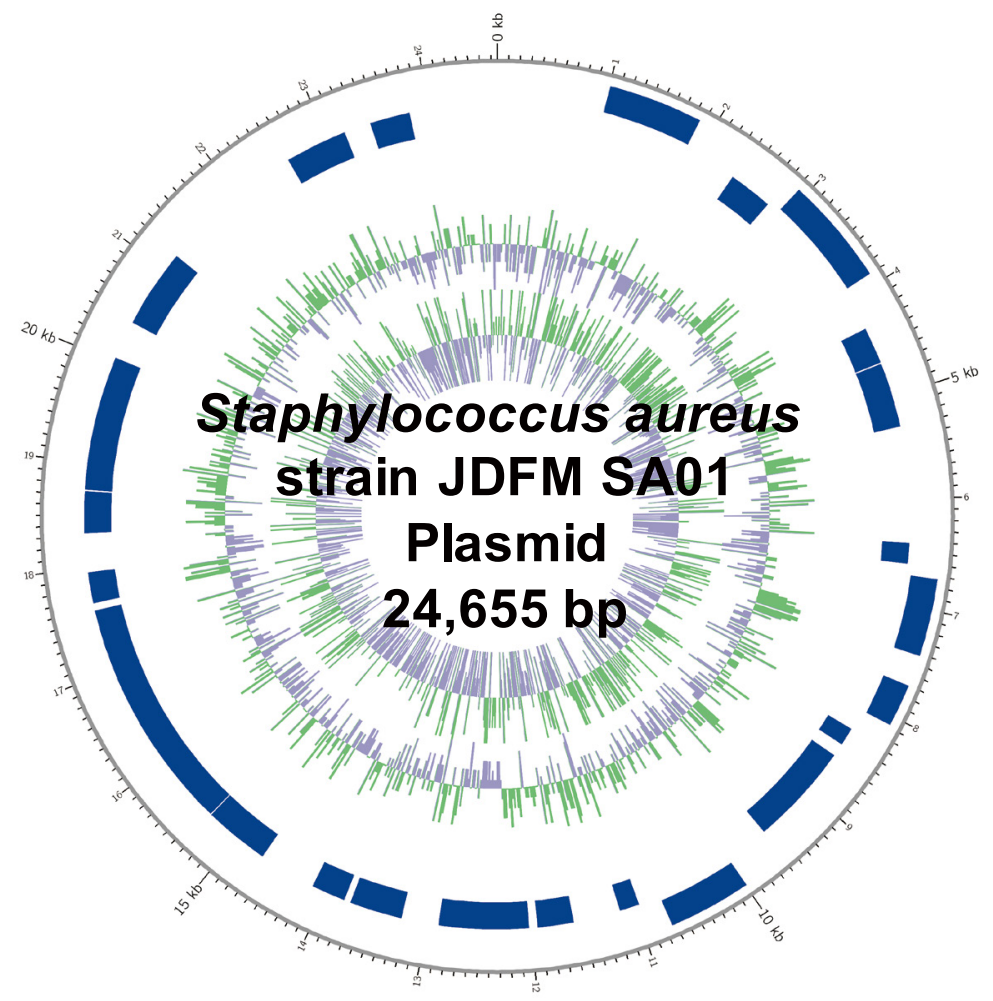

Fig. 1. Circular chromosome and plasmid maps of Staphylococcus aureus strain JDFM SA01. Marked characteristics are shown from outside to the center; coding DNA sequences (CDSs) on forward strand, CDS on reverse strand, tRNA, rRNA, GC content and GC skew. (A) Chromosome, (B) Plasmid. 
Ethics approval and consent to participate This manuscript does not require IRB/IACUC approval. fection by expressing a number of toxic factors, cell wall-related adecines and secreted exoproteins [2]. Therefore, monitoring of $S$. aureus in a dairy environment with high sensitivity is very important for ensuring milk quality and food safety [3].

In the present study, S. aureus strain JDFM SA01 was isolated from a milk filter in Korean dairy farm (Jeollabuk-do; sampled during May 2017 to May 2018). The sample was added to 10\% $\mathrm{NaCl}$-added Tryptic Soy Broth (TSB) and incubated at $37^{\circ} \mathrm{C}$ for $24 \mathrm{~h}$. The enrichment culture was inoculated onto Baird Parker (Oxoid) agar and cultured at $37^{\circ} \mathrm{C}$ for $24 \mathrm{~h}$ [4]. After incubation, a single colony, designated S. aureus strain JDFM SA01, was selected and routinely maintained on tryptic soy broth at $37^{\circ} \mathrm{C}$ for sequencing. Total genomic DNA was extracted using the PureHelix ${ }^{\mathrm{TM}}$ GenomicDNA prep kit (Nanohelix, Korea), according to the manufacturer's instructions.

The whole genome of S. aureus strain JDFM SA01 was sequenced by using the Pacbio RS II (Pacific Biosciences, USA) / Illumina HiSeq (151 × 2 bp paired-end sequencing) platforms at Macrogen (Seoul, Korea). Library preparation for Illumina and PacBio sequencing was performed using the NEBNext Ultra DNA library prep kit for Illumina (NE, USA) and the PacBio DNA template prep kit 1.0 (Pacific Biosciences, USA), respectively, according to the manufacturers' instructions. A total number of 179,260 reads with a mean subread length of 8,498 bases (N50,12,066 bases) were obtained with PacBio sequencing, and 10,257,096 paired-end reads totaling 1,548,821,496 bp were obtained with Illumina sequencing. De novo assembly was carried out using the Hierarchical Genome Assembly Process v3.0 (HGAP3) with default options within SMRT Portal v2.3.0 software [1]. During the preassembly step, filtering and assembly were performed using preAssembler Filter v1 (minimum subread length, $500 \mathrm{bp}$; minimum polymerase read quality, 0.80 ; minimum polymerase read length, $100 \mathrm{bp}$ ) and preAssembler v2 (Minimum seed read length, 6,000 bp; number of seed read chunks, 6; alignment candidates per chunk, 10; total alignment candidates, 24; minimum coverage for correction, 6 ). The read quality was confirmed by aligning shorter reads on longer reads applying Basic Local Alignment with Successive Refinement v1 (BLASR) [5] and correcting errors using Pilon version 1.16 [6]. The chromosome and plasmid annotation performed using rapid prokaryotic genome annotation (Prokka) v1.12b [7].

The complete genome sequence of S. aureus strain JDFM SA01 consists of one circular chromosome $(2,748,925 \mathrm{bp})$ with an overall GC content of $32.9 \%$ and one circular plasmid sequence $(24,655 \mathrm{bp})$ with a GC content of $28.7 \%$. A total of 2,549 predicted genes were identified on the genome, including 19 rRNA, 61 tRNA, and 2,520 coding DNA sequences (CDSs) from chromosome, while 29 CDSs were identified on the plasmid. The genomic information of S. aureus JDFM SA01 could be applied to develop new sanitation strategy for safe and high-quality dairy products.

\section{REFERENCES}

1. Chu J, Vila-Farres X, Inoyama D, Ternei M, Cohen LJ, Gordon EA, et al. Discovery of MRSA active antibiotics using primary sequence from the human microbiome. Nat Chem Biol. 2016;12:1004-6.

2. Rodvold KA, McConeghy KW. Methicillin-resistant staphylococcus aureus therapy: past, present, and future. Clin Infect Dis. 2014;58:S20-7.

3. Yang Q, Zhang Y, Li S, Lu X, Yuan Y, Zhang W. Saltatory rolling circle amplification for sensitive visual detection of Staphylococcus aureus in milk. J Dairy Sci. 2019;102:9702-10.

4. Lee JH, Song KY, Hyeon JY, Hwang IG, Kwak HS, Han JA, et al. Comparison of standard culture method and real-time PCR assay for detection of Staphylococcus aureus in processed and unprocessed foods. Food Sci Anim Resour. 2010;30:410-8.

5. Chaisson MJ, Tesler G. Mapping single molecule sequencing reads using basic local align- 
ment with successive refinement (BLASR): application and theory. BMC Bioinformatics. 2012;13:238.

6. Walker BJ, Abeel T, Shea T, Priest M, Abouelliel A, Sakthikumar S, et al. Pilon: an integrated tool for comprehensive microbial variant detection and genome assembly improvement. PLOS ONE. 2014;9:e112963.

7. Seemann T. Prokka: rapid prokaryotic genome annotation. Bioinformatics. 2014;30:2068-9. 